\title{
Diriger un établissement multiculturel et défavorisé: un défi à part?
}

Stéphanie Bauer, Haute école pédagogique Vaud

Aujourd'hui, les recherches nous montrent que l'école suisse ne permet pas la réussite de tous les élèves et notamment des élèves issus de la migration et des élèves de milieu social défavorisé. L'objectif de cet article est d'interroger le rôle des directions d'établissement scolaire en contexte multiculturel et défavorisé, peu documenté dans la littérature sur la gestion de la diversité culturelle et sur le leadership pédagogique. À travers une étude qualitative-interprétative menée auprès de 13 directions d'établissements multiculturels et défavorisés à Genève, nous donnerons à voir les exigences spécifiques de ce milieu et la manière dont les directions comprennent et agissent en vue de la réussite scolaire de tous les élèves.

Les systèmes éducatifs contemporains ont comme point commun de faire figurer à leur agenda politique l'objectif de la réussite pour tous les élèves. À cette ambition s'ajoute le défi de la diversification socio-culturelle du public scolaire. En effet, dans les centres urbains comme Genève, la diversité culturelle est souvent corrélée à un milieu socio-économique défavorisé (Engel \& LeRoy-Zen Ruffinen, 2015). À l'aide de modèles théoriques du leadership (Leithwood, Seashore Louis, Anderson, \& Wahlstrom, 2004; Theoharis, 2007), cette étude a pour objectif de mettre en lumière la manière dont les directions d'établissements scolaires genevoises conçoivent leur rôle quant à la prise en compte des caractéristiques socio-culturelles des élèves.

\section{Problématique}

Genève est le canton le plus multiculturel de Suisse avec $44 \%$ d'élèves étrangers dans la scolarité obligatoire (Office fédérale de la statistique [OFS], 2017a) et ces élèves sont en majorité issus de milieux sociaux défavorisés (53\%) (Engel \& Le Roy.Zen Ruffinen, 2015). La recherche a mis en évidence depuis un certain temps déjà que les élèves de milieux défavorisés avaient moins de chance de réussir que 
les autres (Hutmacher, 1987). Les enquêtes PISA montrent notamment qu'en Suisse, les élèves de milieux défavorisés ont trois fois plus de risques de faire de mauvaises performances que les élèves de milieux favorisés (Organisation de coopération et de développement économique [OCDE], 2016). Quant aux élèves issus de la migration, la même étude montre qu'ils réussissent moins bien que les élèves autochtones. Ils sont également sur-représentés dans les filières à exigences faibles du secondaire inférieur ainsi que dans l'enseignement spécialisé (OFS, 2017a) et sont trois fois plus touchés par le décrochage scolaire (OFS, 2017b). Dans le canton de Genève, les enquêtes vont dans le même sens en ce qui concerne l'effet du milieu social et de l'origine culturelle (Soussi \& Nidegger, 2017).

Il est important de relever, dans l'idée d'articuler facteur migratoire et social, que toute chose égale par ailleurs, l'origine migratoire a un impact sur la réussite scolaire. Les enquêtes PISA montrent en effet que si l'écart de performance diminue entre élèves autochtones et issus de la migration après contrôle du milieu socio-économique, passant de 60 à 40 points, il reste néanmoins présent (OCDE, 2016). Il en va de même en ce qui concerne les élèves de $2^{e}$ génération pour qui ce même effet perdure (Felouzis, Charmillot, \& Fouquet-Chauprade, 2016).

Si le rôle des enseignant-es dans la problématique de la gestion de la diversité culturelle a été plusieurs fois souligné (Akkari, 2006), le rôle des directions d'établissements scolaires reste peu exploré, notamment en Suisse romande (Bauer \& Akkari, 2015). Pourtant, à l'heure des politiques de la nouvelle gouvernance, ce dernier prend de plus en plus d'importance. En effet, depuis les années 90-2000, les transformations du système éducatif tendent à accroître l'autonomie des établissements scolaires tout en augmentant les attentes en matière de résultats (Bauer \& Perrenoud, 2016; Maulini \& Progin, 2016). À la tête de ces établissements, les directeurs et directrices, accompagné-es de leurs adjoint-es, voient leurs responsabilités et missions renforcées. Dans cette perspective, à Genève, la fonction de directeur-trice d'établissement primaire a été créée en 2008. La création de cette dernière renvoie à un changement de paradigme quant à la manière de considérer la gestion d'établissement scolaire. La fonction se professionnalise (Gather Thurler \& Perrenoud, 2004). Les directions possèdent désormais un statut propre. Elles deviennent garantes de la réussite scolaire des élèves et du bon fonctionnement de l'établissement.

La complexité du métier est bien décrite dans l'étude CADRE qui s'est intéressée au travail réel des directions d'établissements scolaires et sociosanitaires des cantons de Genève et Vaud (Gather Thurler, Kolly Ottiger, Losego, \& Maulini, 2017). Non seulement la quantité de tâches est relevée, mais également la diversité de ces dernières. Le travail est "émietté, mité, régulièrement interrompu, contrarié, empêché» (p. 281). Il est traversé de temporalités diverses (urgence vs. développement à long-terme; calendrier scolaire $v s$. planification de projets, etc.) et de contraintes ambigües, notamment dues à la position d'intermédiaire. La direction est en effet prise entre deux feux, 
celui des injonctions institutionnelles et celui des exigences des collaborateurs et collaboratrices du terrain. Cette posture nécessite de pouvoir négocier habilement avec les forces en présence et de se mouvoir dans le paradoxe et la contradiction (Maulini \& Progin, 2016). La manière dont les directions conçoivent leur rôle est en effet le produit en étroite interaction avec les milieux dans lesquelles elles exercent, ses contraintes institutionnelles ou relationnelles, ainsi que ses propres valeurs et représentations du métier (Maulini, Progin, Jan, \& Tchouala, 2017). Chappuis et Thomas (1995) parle de "rôle subjectif», qui se situe entre le rôle prescrit (les attentes sociales) et le rôle effectif, renvoyant aux pratiques concrètes de «mise en acte» du rôle (p. 21). C'est bien ce rôle subjectif, ou conception du rôle, qui va nous intéresser dans cette étude.

\section{Le Leadership a l'épreuve du milieu multiculturel défavorisé: quelle modélisation?}

Qu'en est-il du rôle spécifique des directions dans la gestion en milieu multiculturel et défavorisé? Pour pouvoir comprendre notre objet, nous allons faire référence à deux cadres théoriques du leadership: le leadership dit pédagogique et celui du leadership pour la justice sociale, en détaillant certaines études empiriques ayant été réalisées dans le domaine.

De manière générale, la plupart des travaux sur le leadership des directions d'établissements scolaires relèvent plutôt des courants de l'école efficace et mettent en avant les bonnes pratiques permettant d'atteindre les objectifs visés (Bauer \& Akkari, 2015). Ces recherches essentiellement quantitatives nous permettent d'identifier certains facteurs de succès comme l'accent mis sur la réussite des élèves (Leithwood et al., 2004), la mobilisation des équipes pédagogiques (Hallinger \& Heck, 1996) et l'instauration d'un climat scolaire propice aux apprentissages (Pelletier, Collerette, \& Turcotte, 2015). Le leadership, génériquement, peut être défini comme le fait de «donner des directions et d'exercer une influence» (Leithwood \& Riehl, 2003, p. 2). Si différentes conceptions du leadership sont distinguées dans la littérature anglo-saxonne, notamment le leadership instructionnel renvoyant aux pratiques d'enseignements ou le leadership transformationnel mettant en avant la capacité d'innovation de l'établissement (Hallinger, 2003), les définitions sont consensuelles quant à la capacité d'influence qui caractérise la personne dirigeante. Cette dimension est également soulignée par les auteurs francophones modélisant un «leadership pédagogique», c'est à dire une capacité d'influence sur les pratiques enseignantes (Progin \& Gather Thurler, 2012). Néanmoins, ces différents modèles ne rendent pas nécessairement explicite la prise en compte des caractéristiques culturelles et sociales des élèves dans un objectif d'équité et de justice sociale.

Un modèle de leadership a attiré notre attention car il permet justement de placer au centre de l'activité dirigeante, la compensation des inégalités et 
la réussite des minorités: le leadership pour la justice sociale. Développé par Thehoaris $(2007,2010)$, il interroge les normes et les pratiques discriminatoires de l'école, sur le plan structurel (filiarisation, composition des classes) ainsi que les attitudes et pratiques des acteurs et actrices impliquées (stéréotypes, préjugés, discrimination). Ce modèle de leadership se distingue en outre par une littérature encore émergente, parfois militante et essentiellement qualitative, là où le leadership pédagogique constitue un corpus de recherche abondant et construit dans une approche plus pragmatique. Par ailleurs, l'opérationnalisation du concept de leadership pour la justice sociale n'est pas très éloignée des dimensions proposées dans la littérature générale sur le leadership, en terme de capacité d'influencer les pratiques à travers une action sur les objectifs de l'établissement, son organisation et ses acteurs internes et ses partenaires externes (Leithwood et al., 2004). Il comporte néanmoins l'intérêt de s'intéresser à un domaine souvent jugé moins important par les directions au regard de questions opérationnelles, administratives ou de ressources humaines (Cattonard et al. 2007; Gélinas Proulx, IsaBelle, \& Meunier, 2014), en portant au cœur de la pratique dirigeante la lutte contre les inégalités.

C'est justement cette interrogation qui était au cœur de l'étude québécoise de Archambault et Harnois $(2009,2010)$ sur la spécificité des écoles en milieu défavorisé et multiculturel. Dans les représentations mobilisées par les directions interrogées quant au rôle à tenir, ces dernières faisaient ressortir les contraintes fortes qu'exerçaient le caractère défavorisé et multiculturel du milieu d'exercice, nuançant ainsi les conclusions de certaines études sur le leadership pédagogique quant aux priorités (à la vision) à donner à l'établissement. Par exemple, l'accent mis sur la performance scolaire des élèves semble moins important que leur bien-être comme condition nécessaire à l'apprentissage. Il en va de même en ce qui concerne les attentes élevées, facteur lié à la réussite. Les directions jugent parfois ces attentes plus «irréalistes» qu'élevées, ce qui amène les auteurs de l'étude à soulever la persistance des représentations négatives associées aux capacités des élèves de milieux défavorisés. Les directions soulignent enfin une charge importante de travail ainsi que la nécessité de travailler à partir d'un engagement moral fort en faveur de la réduction des inégalités et de la justice sociale (Theoharis, 2007, 2010). Ainsi, le milieu multiculturel et défavorisé rend la perception du rôle bien différente d'autres milieux pour les directions qui conçoivent le sens de leur travail, et sa spécificité, dans une prise en compte des besoins spécifiques des élèves fréquentant leur établissement.

Dans cette étude, nous allons voir dans quelle mesure la conception du rôle, abordé dans son sens subjectif (Chappuis \& Thomas, 1995), mobilisée par les directions, intègre ou non une adaptation aux caractéristiques socio-culturelles du milieu, et si oui, de quelle manière. Il apparaît en effet essentiel, au regard des enjeux de réussite scolaire pour les élèves de milieux défavorisés et multiculturels (OCDE, 2016; Soussi \& Nidegger, 2017) et du peu de travaux scientifiques suisses-romands sur la question, de pouvoir mieux comprendre la manière 
dont les directions perçoivent leur fonction. Devant la difficulté du système à faire réussir tous les élèves, la direction d'établissement représente un réel levier d'action. Les contraintes sont néanmoins nombreuses et les stratégies multiples (Gather Thurler et al., 2017). Nous utiliserons le cadre théorique du leadership pour la justice sociale (Theoharis, 2007, 2010) articulé aux facteurs de réussite du leadership pédagogique (Leithwood et al., 2004) comme cadre d'analyse, afin de pouvoir caractériser la façon dont les directions entendent répondre à ce défi. L’opérationnalisation de ces modèles est présentée en annexe.

\section{Méthodologie}

Dans cette étude, nous procéderons à une analyse qualitative-interprétative avec pour objectif de rendre compte du sens que construisent les acteurs sur leur réalité. En d'autres termes, il s'agit à la fois de donner à voir «les positions ontologiques [de l'individu] (sa vision de la réalité) et épistémologiques (associées aux conditions de production du savoir)» (Savoie-Zajc, 2011, p. 176). Dans cette perspective interactionniste, les individus se rattachent à des catégories de pensée pour organiser leur environnement et produire du sens sur les évènements vécus. Ce processus de construction suppose en effet de tenir compte du contexte de production du discours, et de ne pas le considérer comme un objet pré-existant en soi et indépendant de toute interaction.

Nous avons mené 13 entretiens semi-directifs (Blanchet \& Gotman, 2007) auprès de directeurs et directrices de l'enseignement obligatoire genevois primaire et secondaire (cycle d'orientation). ${ }^{1}$ Il s'agit d'un échantillon intentionnel (Savoie-Zajc, 2007). Nous avons choisi de nous intéresser aux établissements multiculturels et défavorisés, à savoir les établissements comptant plus de $40 \%$ d'élèves étrangers et d'élèves de milieux sociaux défavorisés, seuil qui permettait de s'inscrire dans la moyenne cantonale ${ }^{2}$ et d'avoir un nombre suffisant de participants. Neuf directions d'école primaire (six femmes, trois hommes) et quatre directeurs du secondaire ont été interrogés ${ }^{3}$. Les entretiens ont été analysés selon la méthode catégorielle itérative proposée par Miles et Huberman (2003) et à partir du cadre d'analyse construit sur la base des modèles de leadership présenté plus haut (voir annexe). Suivant cette méthode, les données sont d'abord condensées puis organisées et enfin vérifiées, et ce dans un processus circulaire ou «modèle interactif» (Miles \& Huberman, p. 31). La stabilisation des catégories s'est déroulée selon une logique inductive délibératoire (Savoie-Zajc, 2011), où les catégories sont éprouvées à partir d'une construction pré-existante à la récolte de données (dans notre cas, issue des modèles de leadership mobilisés). Cette méthode permet d'aboutir à des catégories d'analyse, nommées indicateurs, sur lesquelles sont construites les questions d'entretien (visibles en annexe). Nous rendrons compte des résultats en faisant ressortir dans les deux premières parties les tendances générales observées dans la majorité des discours ainsi que certaines 
nuances, puis dans la dernière section deux directions qui se distinguent par un engagement plus manifeste envers la réussite scolaire. Nous avons choisi de mettre en évidence ces deux cas «intra-site» (Miles \& Huberman, 2003), également sur la base des entretiens, afin de rendre visible non seulement la singularité de ces discours atypiques mais également l'articulation des différentes dimensions analytiques particulièrement explicites dans ces deux cas.

\section{Analyse des résultats}

\section{Le poids de la défavorisation}

La conception du rôle est-elle influencée par les caractéristiques socio-culturelles des élèves? De manière générale, les directions ont fortement convoqué le marqueur social et beaucoup moins le marqueur culturel. À interroger l'objectif donné au développement de l'établissement au regard des enjeux de réussite scolaire des élèves, il est ressorti assez nettement que la réussite pour tous relève de l'utopie et que l'égalité des chances est un mythe. Certes, elles évoquent des attentes positives envers les élèves issus de la migration quant à leurs capacités scolaires, mais les obstacles restent nombreux sur leur parcours. Les directions soulèvent en effet quasi unanimement le frein trop important que représente le milieu social défavorisé, évoquant même leur impuissance. En particulier, ce sont les conditions de vie matérielles et la capacité des parents à pouvoir suivre la scolarité des enfants qui sont évoquées:

C'est une évidence, quand vous faites appel aux activités parascolaires entre $7 \mathrm{~h}$ le matin et $8 \mathrm{~h}$, et puis que vous êtes rentrés tard le soir, que l'enfant n'a pas fait ces devoirs parce que le parent ne peut plus depuis un certain nombre d'année aider aux devoirs. Je veux dire les contingences matérielles prennent le dessus. (Dir-B)

Si le milieu social joue un rôle effectif sur la réussite comme nous l'avons vu dans la problématique, l'origine culturelle semble en jouer un également, mais cet élément a souvent été réfuté par les directions qui imputent les difficultés uniquement au milieu social. À noter qu'elles donnent presque exclusivement comme exemple les élèves de première génération alors que les chiffres montrent que les élèves de deuxième génération sont également défavorisés à cause de leur seule origine culturelle. Pour ces derniers, les directions ne voient pas forcément de difficulté de réussite: "Ce sont des élèves comme les autres» (Dir-B). Ce problème d'inégalité ne semble donc pas connu des directions, ce qui pourrait se comprendre par le fait d'une invisibilisation de la différence culturelle au profit $\mathrm{du}$ marqueur social, visible également chez les enseignant-es (Akkari, Broyon, \& Changkakoti, 2012). Les politiques cantonales peuvent également être interrogées à la suite de ce constat, car si des ressources sont allouées à partir d'un certain seuil de population défavorisée et allophone, ${ }^{4}$ les réponses proposées à la 
diversité culturelle renvoient essentiellement, en terme de dispositif, à la prise en charge des premières générations (classe d'accueil, soutien à l'apprentissage du français) et moins à la valorisation des identités plurielles ou des langues d'origine. ${ }^{5}$

\section{L'enjeu du projet professionnel}

À la suite d'Archambault et Harnois (2010), l'objectif de réussite scolaire identifiée par la littérature comme un facteur de réussite est fortement nuancé par les directions genevoises, qui le conçoivent moins sous l'angle de la performance que sous celui de l'épanouissement personnel et de l'insertion socio-professionnelle:

Notre mission c'est de permettre à chaque élève de progresser le plus possible, sans que cette progression soit associée à la notion de performance, de progresser dans le développement de sa culture générale [...] Et puis surtout dans la définition de son projet. (Dir-W)

Selon ces directions, ne pas associer la réussite à la performance ne renvoyaient pas à une diminution des attentes mais à leur adaptation à un projet faisant du sens pour l'élève. D'après ce raisonnement, le fait d'étudier dans le regroupement moins exigeant (une caractéristique structurelle de l'organisation scolaire du secondaire 1, accentuant les inégalités selon les enquêtes PISA; OCDE, 2016) n'est pas forcément une impasse. Au contraire, il peut s'agir de la meilleure voie pour l'élève:

Notre but ici dans cette équipe, c'est de se dire "personne n'est laissé de côté». C'est-à-dire que les enfants en sortant de cette école, ils vont au cycle d'orientation. Il y en a moins qui vont dans le regroupement le plus élevé que peut-être dans d'autres écoles. C'est sûr, les chiffres nous le disent. Mais pour nous ce n'est pas un problème. C'est-à-dire que voilà, on n'a pas baissé nos exigences par rapport au plan d'étude. Ils ont plus de peine à atteindre [les objectifs]. On ne vise pas le 6 , ni même le 5 par exemple. On se dit ben voilà il faut qu'ils soient bien dans leur métier d'élève, qu'ils aient un projet de vie, ils passent au cycle d'orientation. Ce que je sais c'est que quand ils y sont, ils sont bien orientés. (Dir- A)

L'accent mis sur l'épanouissement de l'élève est intéressant de deux façons. Il permet de s'interroger dans un premier temps sur le sens que les directions donnent à la réussite scolaire et nous voyons ici que la vision n'est pas uniquement axée sur les performances. La question de la réussite n'est pas une question structurelle mais une question de sens par rapport aux finalités de l'école. Filière ou pas filière, que faire avec ces élèves pour qui l'enseignement supérieur n'est pas la voie du succès? Comment les aider à construire un projet professionnel porteur? C'est ici que la mobilisation de l'épanouissement ou du bien-être par les directions représente un regard pertinent sur le problème, dans la mesure où il met l'accent sur l'intégration professionnelle de l'élève plutôt que sa performance académique, souvent seul critère mesurant la réussite d'un élève. D'un autre côté, 
on peut y voir une explication de la persistance des inégalités dans la mesure où le système est considéré comme fonctionnant, il n'est pas remis en question, et les attentes envers ces élèves ne sont pas élevées.

L'exercice du leadership en milieu multiculturel et défavorisé amène les directions à relativiser l'importance donné à la performance scolaire dans la vision de la réussite des élèves, au profit d'une définition autour du bien-être, comme l'ont montré Archambault et Harnois (2010), pour qui le sens de l'engagement pour la justice sociale passe par cette préoccupation. Par ailleurs, au regard du modèle de leadership pour la justice sociale, la remise en question des barrières structurelles que représenteraient ici les filières de sélection pour les élèves issus de la migration et de milieux défavorisés n'est pas retenue comme un facteur influençant la conception de leur rôle. Ce constat peut être mis en parallèle avec l'invisibilisation de la dimension culturelle soulignée plus haut, mais également révéler la limite de l'utilisation d'un modèle nord-américain, construit dans un contexte où la justice sociale et la correction des inégalités possède une ampleur plus grande dans le discours socio-politique qu'en Suisse (Akkari, 2009).

\section{La diversité socio-culturelle au centre de \\ l'engagement collectif}

Deux directeurs sortaient du lot dans notre série d'entretien, celui de l'école primaire $\mathrm{F}$ et de l'établissement secondaire Y. Leur conception du rôle présentait la particularité d'intégrer la prise en compte de la diversité culturelle et sociale dans tous les domaines d'action de leur métier: objectif de développement et organisation de l'établissement, pratiques pédagogiques et relations aux partenaires scolaires.

Le discours du directeur de l'établissement $Y$ se distinguait par une mise en évidence de l'engagement collectif des enseignant-es quant à la pertinence de leur pratique pédagogique au regard du public scolaire. Le directeur concevait ainsi son rôle comme un "motivateur» des équipes pédagogiques dans un processus de renouvellement, insufflé il y a quelques années devant le constat de l'échec des modes d'enseignement usités. La direction et l'équipe pédagogique ont fixé certaines priorités quant à la prise en compte de la vulnérabilité des élèves face à l'échec scolaire. Ils ont construit une pédagogie autour du respect du processus d'apprentissage, et notamment du «moment de déséquilibre affectif et cognitif, où on est extrêmement fragile» en imposant un cadre plus strict où plus aucune moquerie n'était tolérée. L'importance donnée au climat d'apprentissage sûr illustre les facteurs de réussite soulevés par Pelletier et al. (2015) et ne se distingue en soi pas forcément d'un leadership pédagogique axé sur les apprentissages et la réussite des élèves. Toutefois, nous pouvons articuler ce constat avec l'emphase portée au bien-être des élèves (Archambault $\&$ Harnois, 2010) et à la nécessité de porter en priorité l'établissement de conditions d'apprentissage favorables, et ce d'autant plus que des conditions défavorables ont un impact fort sur les élèves les plus vulnérables. 
La prise en compte des caractéristiques culturelles des élèves se manifestait tout particulièrement dans la manière dont les directions concevaient leurs relations aux familles. Si les marqueurs sociaux et culturels se distinguaient peu dans les conceptions générales des directions, la relation aux familles permettait de faire ressortir plus clairement la dimension (inter)culturelle de cette relation. Les directions $\mathrm{Y}$ et $\mathrm{F}$ insistaient en effet sur la nécessité de prendre plus de temps pour accueillir les familles issues de la migration. Dans le cas de l'établissement $\mathrm{Y}$, les entretiens avaient pour but de créer une relation de confiance en montrant notamment que le but commun était l'intérêt de l'élève. L'objectif n'en était pas pour autant de nier la différence culturelle. Le directeur explique dans cet extrait la nécessité de pouvoir tenir compte de la dualité du rapport à l'intégration des familles:

Il y a toujours cette tension de l'étranger à d'une part vouloir être traité comme tout le monde, ce qui serait pour lui une preuve qu'il est assimilé, c'est quand même un but qu'il recherche, et puis aussi sa volonté forte de dire: mais moi je suis porteur d'une différence et j'aimerais bien qu'on l'identifie. (Dir-Y)

L'enjeu des rencontres était donc de rendre explicite le respect pour la différence tout en montrant le dénominateur commun, comme par exemple des valeurs de respect, de bienveillance ou de travail. Les caractéristiques culturelles de familles, qu'elles soient religieuses, linguistiques, ethniques, étaient tout autant de paramètre à considérer afin de construire un dialogue. Les directions concevaient leur rôle comme celui d'un partenaire où la valeur de chaque partie est reconnue de manière égale (Vatz Laaroussi, Kanouté, \& Rachédi, 2008). Nous avons constaté le même discours dans le cas de l'école F.

Le discours du directeur de l'école primaire $F$ se détachait également des autres quant à la manière dont les caractéristiques socio-culturelles des élèves constituaient le point de départ de toute action pédagogique et s'intégrait à la définition de l'identité de l'établissement. Comme le directeur Y, le directeur F mettait en avant son rôle de soutien au travail en équipe des enseignant-es, et celui de partenaire vis à vis des familles. Il se distinguait de son prédécesseur cependant en remettant plus explicitement en cause le caractère ethnocentrique et discriminatoire de l'organisation scolaire:

On sait que l'école provoque, entretient les inégalités de chance, parce que tout ça, c'est assez rigide quand même. Ces fiches, ces machins auxquels il faut répondre, il n'y a qu'une façon de répondre. Et puis après vous allez dans une classe, et vous vous rendez compte que l'enseignant, il est comme les fiches. (Dir-F)

Le directeur interroge plus précisément les représentations des enseignant-es dans leur rapport à l'altérité. Au-delà des jugements négatifs entravant la réussite scolaire, relevés par les directions et étayé par Bressoux et Pansu (2003), c'est la question de l'engagement collectif qui est soulevée. La mobilisation des équipes est en effet identifiée comme étant un facteur-clé du renouveau pédagogique 
et donc, de la réussite scolaire (Leithwood et al., 2004). Elle est ici au service de la prise en compte de la diversité culturelle et sociale des élèves, dans un objectif de compensation des inégalités et de justice sociale (Theoharis, 2007, 2010). L'adaptation aux caractéristiques du public scolaire n'est plus de la seule responsabilité de l'enseignant-e dans sa classe mais celle de l'établissement en entier et la collaboration professionnelle devient alors plus évidente.

Dans ce sens, l'école $\mathrm{F}$ a pu concrétiser cette dynamique à travers un projet qui rassemblait non seulement les enseignant-es mais également les familles et la communauté: l'école des mamans. Des cours de français étaient proposés aux mamans d'élèves dans les locaux scolaires, afin de faciliter le suivi de la scolarité de leurs enfants: "On leur apprend à s'intégrer, à travers l'importance de parler français pour aider leurs enfants» (Dir-F). Les mamans rémunèrent ce service en amenant des repas pour les enseignant-es, leur permettant ainsi de pouvoir participer plus facilement à la vie de l'école. L'éducatrice de l'école ainsi que la commune ont fait partie du projet qui a rencontré un franc succès. Il a notamment donné lieu à la participation des mamans dans les classes via des activités de type EOLE (ouverture aux langues) faisant ainsi profiter l'ensemble des élèves tout en décloisonnant l'organisation scolaire habituelle (Changkakoti \& Hutter, 2015).

Dans le processus de construction du projet, la question des valeurs était au centre des négociations menées avec les familles comme pour l'établissement Y. Ce changement de représentation a été radical notamment dans l'accueil qui mobilisait la direction, les enseignants et l'éducatrice à travers un "protocole très clair d'accueil: on prend le temps» (Dir-F). Toutes les premières étapes du contact avec les familles ont été repensées afin de sortir d'une posture injonctive «elles n'ont qu'à s'intégrer» et de pouvoir entrer dans une co-construction de référents communs. Ce qui a facilité ce travail, selon le directeur, était la collaboration étroite avec la communauté 6 , plus particulièrement l'association de quartier, qui permettait d'élargir les possibilités d'accueil et de dialogue avec les familles (hors temps scolaire par exemple). C'était le seul cas de notre corpus qui relatait cette collaboration. Du côté de la gestion des équipes enseignantes, il était nécessaire, pour le directeur, que tous adhèrent à ce principe de reconnaissance de la diversité. C'était une condition exprimée au moment du recrutement du personnel et lors des discussions collectives autour des projets d'établissement. Nous pouvons ainsi remarquer une nouvelle fois la prépondérance de la question du pilotage des équipes enseignantes dans la pratique du leadership (Leithwood et al., 2004) ainsi que la place majeure donnée à la diversité culturelle dans la définition de l'action pédagogique et de l'organisation scolaire (Thehoaris, 2007, 2010).

Nous avons donc pu constater que les établissements $\mathrm{Y}$ et $\mathrm{F}$ se distinguaient des autres par des mesures concrètes de prise en compte de la diversité. Les deux directeurs concernés ont rendu explicite la manière dont les caractéristiques socioculturelles des élèves influençaient leur travail au quotidien, à travers la mise en évidence du «rôle réel» que demandait l'adaptation aux besoins du public scolaire. 
Au-delà du discours consensuel sur la richesse de la diversité, ils pouvaient relater des pratiques pédagogiques différentes intégrant le facteur défavorisation ainsi que des pratiques de négociation et de communication interculturelle avec les familles et la communauté. Le point de départ de ce changement, la "claque», où le constat de l'inefficacité des pratiques existantes, a donné lieu à une remise en question des normes scolaires entravant la réussite scolaire des élèves issus de la migration. Cette démarche s'inscrit dans l'objectif central du leadership pour la justice sociale (Theoharis, 2007) et rejoint les conclusions d'Archambault et Harnois (2010) quant à la reconnaissance des besoins spécifiques du milieu scolaire. La reconstruction s'est opérée avec une mise en évidence des valeurs permettant de respecter les différences et de rassembler les individus sous une vision commune.

\section{Conclusion}

Ainsi, de manière générale, il semblerait que les caractéristiques socio-culturelles des élèves ne représentent pas un critère majeur permettant de comprendre des variations dans la conception du rôle que construisent les directions. Nous pouvons relever deux nuances à ce constat. La première renvoie aux discours des directions $\mathrm{Y}$ et $\mathrm{F}$ qui, contrairement à la tendance générale, exposent un engagement en faveur d'une réduction des inégalités et intègrent la prise en compte des besoins des familles et élèves dans la manière de diriger l'établissement en mobilisant l'ensemble des domaines d'action du leadership pour la justice sociale (Theoharis, 2010). L'engagement collectif de l'équipe pédagogique représente notamment un levier d'action important et encouragé par les directions. Il facilite la collaboration et l'adaptation des pratiques aux caractéristiques socioculturelles des élèves et de leur famille. Ensuite, la manière dont la notion de réussite est repensée chez les directions œuvrant en milieu multiculturel et défavorisé, autour du bien-être plutôt que de la performance, et de l'importance d'un climat favorable aux apprentissages, est un constat similaire à celui formulé par Archambault et Harnois (2010). Ces résultats concordants nous permettent de souligner la nécessité de remettre en question la vision unidimensionnelle de la réussite comme performance de manière plus large, en s'inscrivant dans une conception plus holistique de l'expérience scolaire (Ryan \& Laguardia, 2000).

Par ailleurs, il convient de relever la limite de la portée des modèles théoriques $\mathrm{du}$ leadership essentiellement anglo-saxons pour notre contexte d'étude. Si la manière dont ils conceptualisent le rôle des directions à travers la mise en évidence de différents domaines d'action semble pertinent au regard de nos résultats, le rapport à la diversité socio-culturelle et à la justice sociale ne résonne pas de la même manière à Genève où les modèles socio-politiques de gestion de la diversité, a fortiori culturelle, mettent moins l'accent sur la compensation des inégalités que sur l'intégration des étrangers (Akkari, 2009). Ainsi, la conception 
du leader engagé contre les injustices ne représente pas un modèle très vulgarisé en Suisse romande. Le concept même de leadership peut susciter de la méfiance auprès des directions qui le considèrent comme trop personnifié ou renvoyant à des relations conflictuelles (Progin \& Perrenoud, 2018). Néanmoins, les résultats de notre étude permettent de mieux définir la manière dont les directions se représentent leur fonction au regard des objectifs de réussite scolaire et de lutte contre les inégalités dans un contexte de diversité socio-culturelle croissante.

Enfin, si l'autonomie des établissements et le renforcement des pouvoirs des directions participent à ces réformes de la nouvelle gouvernance, la marge de manœuvre des directions n'en est pour autant pas infinie. Outre les contraintes de l'organisation scolaire et des plans d'études, la charge administrative et les relations avec le personnel éducatif sont tout autant de préoccupations quotidiennes qui ralentissent la capacité d'innovation de l'établissement (Gather Thurler et al., 2017). Par ailleurs, les politiques genevoises de prise en compte de la diversité socio-culturelle ne proposent pas un cadre de référence très explicite quant aux réponses à apporter. L'autonomie prônée laisse ici les directions devant une absence de réponse concrète face au problème des inégalités. Une ouverture possible amenée par nos résultats semble celle des projets d'établissements qui permettent à la direction, à l'équipe enseignante ainsi qu'aux partenaires externes de pouvoir proposer des objectifs et des moyens d'action en adéquation avec les besoins du public fréquentant l'école. Au regard de nos résultats et des premières évaluations conduites de ce dispositif (Gros, Guilley, Jaeggi, Sermet, \& Arnold, 2012), il semblerait que les projets représentent une piste intéressante à suivre.

\section{Notes}

1 Les entretiens ont été enregistrés avec un enregistreur audio, puis retranscrits intégralement. Ils ont duré en moyenne 105 minutes et ont été analysés à l'aide du logiciel d'analyse N-Vivo, dans le cadre d'une recherche doctorale (2010-2016).

2 Le taux moyen d'élèves étrangers par classe dans le canton est de 44\% (OFS, 2017) et celui d'élèves défavorisés est d'environ 38\% (Engel \& Le Roy-Zen Ruffinen, 2015).

3 Les participants seront désignés par un code lié à leur école: A à I pour le primaire et W à $\mathrm{Z}$ pour le secondaire.

4 Le «statut école» en réseau éducatif prioritaire (REP) est accordé aux établissements dont la population dépasse $55 \%$ d'élèves de milieux défavorisés et $40 \%$ d'élèves allophones. Il donne notamment droit à un nombre plus faible d'élève par classe, un-e éducateur-trice et une enveloppe budgétaire plus élevée.

5 Des cours de langue et culture d'origine sont proposés aux élèves dans les locaux scolaires mais sont organisés par des associations ou institutions des pays d'origine.

6 La communauté représente ici la communauté éducative élargie, c'est-à-dire l'ensemble des partenaires professionnels et associatifs réunis autour de l'école. 


\section{Références}

Akkari, A. (2006). Les approches multiculturelles dans la formation des enseignants: entre recherche et pédagogie critique. Revue des HEP de la Suisse romande et du Tessin, 4, 233-258.

Akkari, A. (2009). Introduction aux approches interculturelles en éducation. Genève, Suisse: Université de Genève.

Akkari, A., Broyon, M. A., \& Changkakoti, N. (2012). Les enseignants et la diversité culturelle en Suisse: entre rhétorique de valorisation et réalités du terrain. Ville-École-Intégration Diversité, 168, 103-110.

Archambault, J., \& Harnois, L. (2009). Diriger une école primaire de milieu urbain défavorisé: la justice sociale au coeur du travail de directions d'école. Ethique publique, 11(1), 86-93.

Archambault, J., \& Harnois, L. (2010). Les réactions de directions d'écoles de milieux défavorisés aux caractéristiques des écoles performantes de milieux défavorisés. Nouveaux cahiers de la recherche en éducation, 13(2), 109-126.

Bauer, S., \& Akkari, A. (2015). La gestion de la diversité culturelle par les chefs d'établissement: une revue de la littérature. Revista Educação e Políticas em Debate, 4(1), 150-172.

Bauer, S., \& Perrenoud, O. (2016). Nouveaux modes de gouvernance et pilotage des établissements scolaires en Suisse romande. Formation et profession, 24(1), 84-86.

Blanchet, A., \& Gotman, A. (2007). L'enquête et ses méthodes: l'entretien. Paris, France: Armand Colin.

Bressoux, P., \& Pansu, P. (2003). Quand les enseignants jugent leurs élèves. Paris, France: PUF.

Cattonar, B., Lessard, C., Blais, J.-G., Larose, F., Riopel, M.-C., Tardif, M., Bourque, J., \& Wright, A. (2007). Les directeurs et les directrices d'école au Canada: contexte, profil et travail. Enquêtes pancanadiennes auprès des directions et des enseignants d'écoles primaires et secondaires (2005-2006). Canada: Chaire de recherche du Canada sur le personnel et les métiers de l'Éducation.

Changkakoti, N., \& Hutter, V. (2015). L'école des mamans et autres histoires d'accueil: une expérience d'histoire inclusive. In A. Manço (Éd.), Pratiques pour une école inclusive: agir ensemble (pp. 157-169). Paris, France: L’Harmattan.

Chappuis, R., \& Thomas, R. (1995). Rôle et statut. Paris, France: PUF.

Engel, B., \& Le Roy.Zen Ruffinen, O. (2015). L'enseignement à Genève. Repères et indicateurs statistiques. Genève, Suisse: SRED.

Felouzis, G., Charmillot, S., \& Fouquet-Chauprade, B. (2016). Les élèves de deuxième génération en Suisse: modes d'intégration scolaire et compétences acquises dans 13 systèmes éducatifs cantonaux. Revue suisse de sociologie, 42(2), 218-244.

Gather Thurler, M., \& Perrenoud, P. (2004). Professionnalisation et formation des chefs d'établissement. Administration et éducation, 102, 67-76.

Gather Thurler M., Kolly Ottiger I., Losego P. \& Maulini O. (2017). Les directeurs au travail. Une enquête au cour des établissements scolaires et socio-sanitaires. Berne, Suisse: Peter Lang.

Gélinas Proulx, A., IsaBelle, C., \& Meunier, H. (2014). Compétence des nouvelles directions d'école de langue française au Canada pour la gestion inclusive de la diversité ethnoculturelle, linguistique et religieuse. Alterstice-Revue Internationale de la Recherche Interculturelle, 4(1), 73-88.

Gros, D., Guilley, E., Jaeggi, J-M., \& Sermet, G. (collab. Arnold, P.). (2012). Evaluation externe des projets d'établissement de l'enseignement primaire: rapport de synthèse. Genève, Suisse: SRED.

Hallinger, P. (2003). Leading educational change: Reflections on the practice of instructional and transformational leadership. Cambridge Journal of education, 33(3).

Hallinger, P. \& Heck, R. H. (1996). The principal's role in school effectiveness: An assessment of methodological progress, 1980-1995. In K. Leithwood, D. Chapman, P. Corson, P. Hallinger et A. Hart (Eds.), International handbook of educational leadership and administration (pp. 723-783). Dortrecht, Pays-Bas: Springer.

Hutmacher, W. (1987). Le passeport ou la position sociale? Quelques données sur la réussite et 
l'orientation scolaire d'enfants suisses et étrangers, compte-tenu de la position sociale de leur famille. Paris, France: OCDE.

Leithwood, K. A., \& Riehl, C. (2003). What we know about successful school leadership. Philadelphia, PA: Temple University.

Leithwood, K., Seashore Louis, K., Anderson, S., \& Wahlstrom, K. (2004). Review of research: How leadership influences student learning. Consulté le 20 juillet 2017 dans http://www. wallacefoundation.org/knowledge-center/school-leadership/key-research/documents/ how-leadership-influences-student-learning.pdf

Maulini, O., \& Progin, L. (2016). Des établissements scolaires autonomes? Entre inventivité des acteurs et éclatement du système. Paris, France: ESF

Maulini, O., Progin, L., Jan, A., \& Tchouala, C. (2017). Sous le travail réel: la conception du rôle et le travail espéré. In M. Gather Thurler, I. Kolly Ottiger, P. Losego, \& O. Maulini (Éds.), Les directeurs au travail. Une enquête au cour des établissements scolaires et sociosanitaires (pp. 109-160). Berne, Suisse: Peter Lang.

Miles, M. B., \& Huberman, A. M. (2003). Analyse des données qualitatives: Méthodes en sciences humaines. Bruxelles, Belgique: De Boeck.

OCDE. (2016). PISA, 2015 Results: Excellence and Equity in Education (vol. 1). Paris, France: OCDE.

OFS. (2017a). Statistiques des élèves et des étudiants. Consulté le 15 septembre 2017 dans https://www.bfs.admin.ch/bfs/fr/home/statistiques/education-science/personnesformation/ecole-obligatoire.html

OFS. (2017b). Statistiques des élèves et des étudiants. Consulté le 15 septembre 2017 dans https://www.bfs.admin.ch/bfs/fr/home/statistiques/education-science/indicateursformation/indicateurs/decrochage-scolaire.html

Pelletier, D., Collerette, P., \& Turcotte, G. (2015). Les pratiques de gestion des directions d'école secondaire sont-elles liées à la réussite des élèves? Canadian Journal of Education/ Revue canadienne de l'éducation, 38(1), 1-23.

Progin, L., \& Gather Thurler, M. (2012). Le leadership pédagogique: un levier pour transformer l'organisation du travail au sein des établissements scolaires. Recherches en éducation, 4, 42-54.

Progin, L., \& Perrenoud, O. (2018). Le leadership: un processus distribué au sein des établissements scolaires? Le cas de la Suisse francophone. Education et francophonie, 46, 33-49.

Ryan, R. M. \& Laguardia, J. (2000). Buts personnels, besoins fondamentaux et bien-être: théorie de l'auto-détermination et application. Revue québécoise de psychologie, 21(2), 281-304.

Savoie-Zajc, L. (2007). Comment peut-on construire un échantillonnage scientifiquement valide? Recherches qualitatives, 99-111.

Savoie-Zajc, L. (2011). La recherche qualitative/interprétative en éducation. In T.Karsenti \& L. Savoie-Zajc (Éds.), La recherche en éducation (pp. 123-147). Québec: éditions du renouveau pédagogique.

Soussi, A., \& Nidegger, C. (2017). Comparaison de deux types d'évaluation externe (PISA et évaluation commune genevoise) en mathématiques et en sciences: compétences mesurées et résultats des élèves. Genève, Suisse: SRED.

Theoharis, G. (2007). Social justice educational leaders and resistance: Toward a theory of social justice leadership. Educational Administration Quarterly, 43(2), 221.

Theoharis, G. (2010). Disrupting injustice: Principals narrate the strategies they use to improve their schools and advance social justice. The Teachers College Record, 112(1).

Vatz Laaroussi, M., Kanouté, F., \& Rachédi, L. (2008). Les divers modèles de collaborations familles immigrantes-écoles: de l'implication assignée au partenariat. Revue des sciences de l'éducation, 34(2), 291-311. 
Mots-clés: Leadership pédagogique, leadership pour la justice sociale, directions d'établissement, diversité culturelle, défavorisation.

\section{Stehen Schulleiter/innen in multikulturellen und Schulen mit benachteiligter Schülerschaft vor besonderen Herausforderungen?}

\section{Zusammenfassung}

Die Schweizer Schulen sind multikultureller als zuvor, insbesondere in städtischen Gebieten. Eines der Hauptprobleme im Zusammenhang mit der beobachteten Vielfalt in der Schule besteht in der Gewährleistung von Gerechtigkeit und Erfolg für alle Schüler/innen. Die Forschung zeigt, dass Schüler/innen mit Migrationshintergrund und niedrigem sozioökonomischem Status im Vergleich zu anderen Schüler/innen ein höheres Risiko für Schulversagen aufweisen. Im Gegensatz zu den Lehrern/innen wurde der Rolle der Schulleiter/innen bezüglich der Frage der Gerechtigkeit weniger Aufmerksamkeit geschenkt. Ziel dieses Beitrags ist es, die spezifische Rolle von Schulleitern in einem multikulturellen und benachteiligten Schulkontext zu untersuchen. Zu diesem Zweck wurde eine qualitative Analyse in Genf mit 13 Schulleitern durchgeführt. Die Ergebnisse zeigen, wie spezifische Herausforderungen multikultureller Schulen mit benachteiligter Schülerschaft von Schulleitern wahrgenommen, verstanden und bei Entscheidungen berücksichtigt werden.

Schlagworte: Pädagogischer Leadership, soziale Gerechtigkeit, Schulleitungen, kulturelle Vielfalt, benachteiligte Schulen

\section{I dirigenti scolastici nelle scuole multiculturali e svantaggiate devono affrontare sfide specifiche?}

\section{Riassunto}

Le scuole tendono ad essere più multiculturali rispetto a prima, specialmente nei centri urbani. Uno dei principali problemi legati alla diversità a scuola è garantire equità e successo per tutti. La ricerca mostra che gli studenti con un background di immigrati e basso status socio-economico hanno maggiori probabilità di rischiare un fallimento scolastico. Mentre il ruolo degli insegnanti nella promozione dell'equità è stato descritto in letteratura per molti anni, poca attenzione è stata rivolta ai dirigenti scolastici. Lo scopo di questo articolo è quello di affrontare il ruolo specifico dei dirigenti scolastici in un contesto multiculturale e svantaggiato. Un'analisi qualitativa è stata condotta a Ginevra, in Svizzera, con 13 dirigenti scolastici. I risultati mostrano come le sfide specifiche delle scuole multiculturali e svantaggiate siano comprese e tenute in considerazione dai dirigenti scolastici. 
Parola chiave: Direzione scolastica, leadership per la giustizia sociale, diversità culturale, scuola svantaggiata, dirigenti scolastici.

\section{Do school principals in multicultural and disadvantaged schools face specific challenges?}

\section{Summary}

Schools tend to be more multicultural than before, especially in urban centers. One of the main issues related to diversity in school is ensuring equity and success for all. Research shows that students with an immigrant background and low socio-economical status are more likely to risk school failure. Whereas the role of teachers on promoting equity has been described in literature for many years, little attention has been paid to school principals. The aim of this paper is to address the specific role of school principals in a multicultural and disadvantaged context. A qualitative analysis was conducted in Geneva, Switzerland with 13 principals. Findings show how specific challenges from multicultural and disadvantaged schools are understood and taken into account by school principals.

Keywords: School leadership, leadership for social justice, school principals, cultural diversity, disadvantaged schools.

Stéphanie Bauer, Dr. est chargée d'enseignement à la Haute école pédagogique du canton de Vaud. Ses recherches s'intéressent au leadership pour la justice sociale ainsi qu'aux approches interculturelles et inclusives en éducation. Elle intervient dans la formation initiale et continue des enseignant-es et co-dirige un groupe de recherche au sein du Laboratoire international sur l'inclusion scolaire (LISIS).

Haute école pédagogique du canton de Vaud, Avenue des Bains 21 - B21-634, $\mathrm{CH}-1014$ Lausanne

E-Mail: Stephanie.bauer@hepl.ch 


\begin{tabular}{|c|c|c|c|c|}
\hline 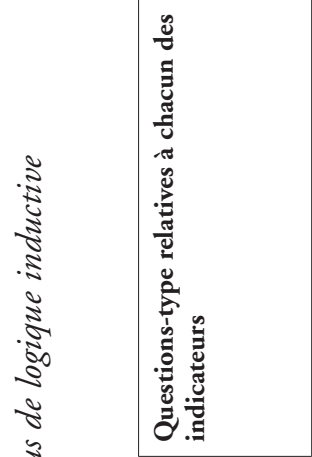 & 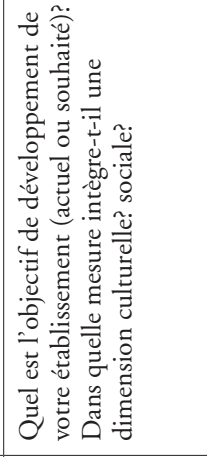 & 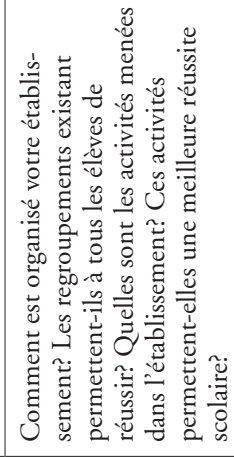 & 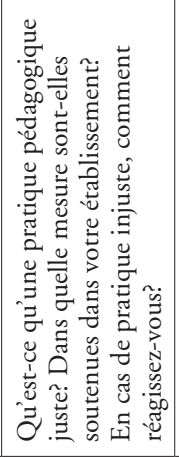 & 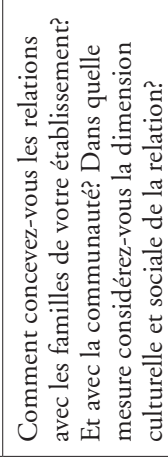 \\
\hline 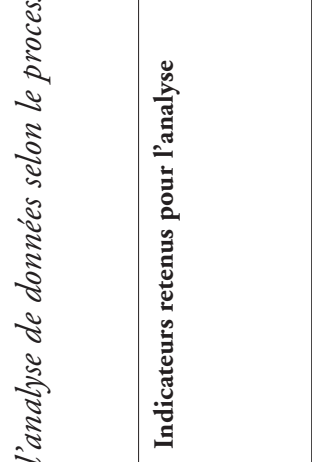 & 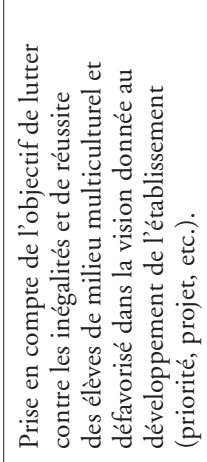 & 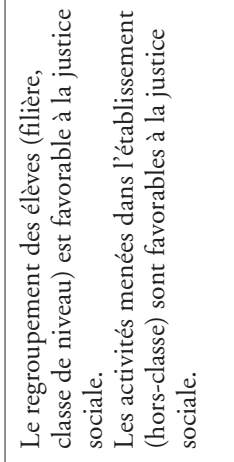 & 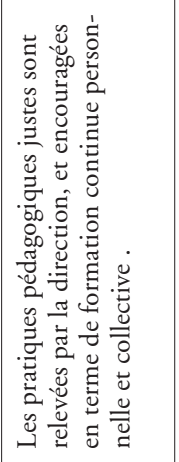 & 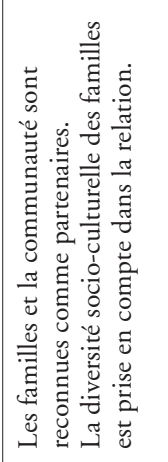 \\
\hline 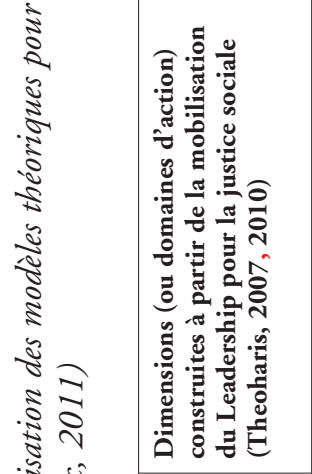 & 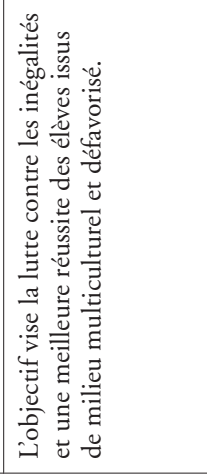 & 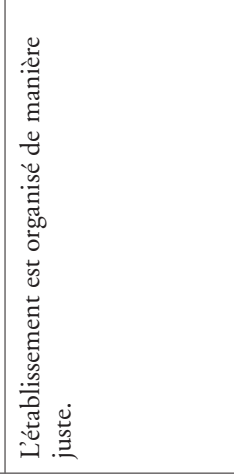 & 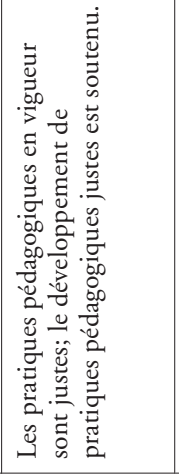 & 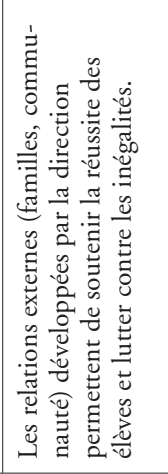 \\
\hline 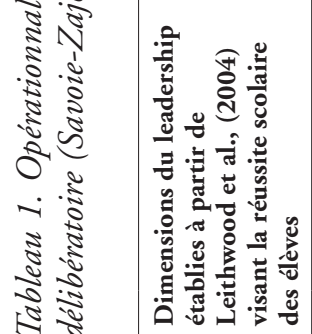 & 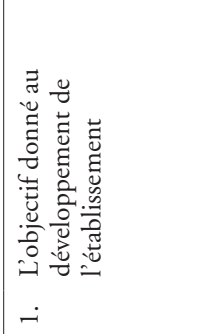 & 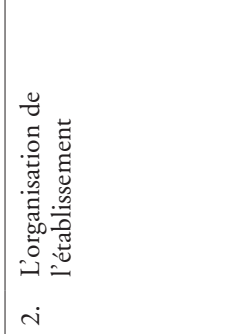 & 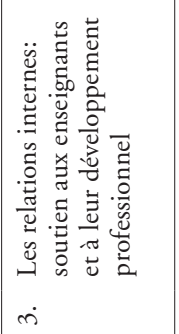 & 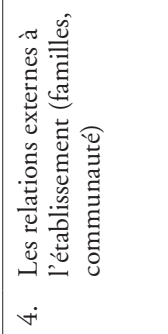 \\
\hline
\end{tabular}

